
éducation relative à l'environnement

\title{
Un « patrimoine » de recherche en construction
}

Lucie Sauvé

\section{(2) OpenEdition \\ Journals}

Édition électronique

URL : https://journals.openedition.org/ere/7140

DOI : $10.4000 /$ ere. 7140

ISSN : 2561-2271

Éditeur

Centr'ERE

Référence électronique

Lucie Sauvé, «Un « patrimoine » de recherche en construction », Éducation relative à l'environnement [En ligne], Volume 1 | 1999, mis en ligne le 15 septembre 1999, consulté le 28 mai 2021. URL : http://

journals.openedition.org/ere/7140; DOI : https://doi.org/10.4000/ere.7140 


\title{
Un « patrimoine » de recherche en construction
}

\author{
Lucie Sauvé
}

1 L'organisation du Colloque international francophone La recherche en éducation relative à l'environnement : bilan, enjeux et perspectives, tenu à Montréal, les 5 et 6 novembre $1997^{1}$, a donné lieu à un véritable processus de recherche en lui-même. Au départ, il a fallu repérer les acteurs d'une telle recherche et identifier leur milieu d'appartenance professionnelle, de façon à solliciter leur participation. Puis, nous avons entrepris une exploration des productions de recherche en ce domaine dans le but de préparer et de stimuler la discussion sur le bilan, les enjeux et les perspectives de développement de la recherche en ERE. L'organisation du colloque a donc impliqué l'amorce de la réalisation d'un Répertoire de la recherche francophone en éducation relative à l'environnement (ERE), élaboré dans une perspective descriptive et critique (voir la section Répertoire de ce volume).

2 Des questions fondamentales ont émergé au cours de cette étape préparatoire qui a impliqué également l'analyse des propositions de communication en vue de les reconnaitre (ou non) comme se rattachant au domaine de la recherche en ERE. Par exemple : Quelles sont les caractéristiques spécifiques d'une recherche en ERE ? Qu'estce qui distingue recherche et intervention en ERE ? À quelles conditions un exercice de réflexion sur l'ERE peut-il devenir une véritable activité de recherche?

D'autres questions ont été associées à l'exploration sommaire des productions de recherche et ont donné lieu à des discussions lors des activités du colloque. Par exemple: Comment se caractérise la recherche en ERE? Quels types de recherche y retrouve-t-on? Quels sont les objets de recherche privilégiés? Quelle est la pertinence éducationnelle et sociale de la recherche actuelle en ERE? Comment renforcer cette double pertinence?

4 Dans les pages qui suivent, nous esquisserons quelques éléments de réponse à ces questions. Nous soumettons ces réflexions et observations aux acteurs de la recherche en ERE, afin de stimuler la discussion. 


\section{À quoi peut-on reconnaître qu'une activité de recherche s'inscrit dans le champ spécifique de la recherche en ERE ?}

5 Cette question nous ramène inévitablement au choix d'une définition de l'éducation relative à l'environnement. Dans la pluralité des courants et des théories qui enrichissent le domaine de l'ERE (Sauvé, 1997 a), quelle définition apparaît la plus apte à cerner le domaine, sans le rétrécir ni le confondre dans un ensemble trop vaste ? Plutôt que d'adopter une définition fermée, nous avons opté pour une caractérisation de l'ERE qui puisse convenir à une pluralité de conceptions spécifiques complémentaires.

6 L'ERE est donc considérée ici comme cette dimension de l'éducation contemporaine qui se préoccupe de reconstruire le réseau des relations personne - groupe social environnement (Sauvé, 1997 a). Dans cette perspective, l'environnement est un écosociosystème, selon l'expression de Louis Goffin (1998), caractérisé par l'interaction entre ses composantes biophysiques et sociales; les deux types de composantes sont nécessairement présents au sein d'une question dite environnementale. Par conséquent, une intervention éducative ou une recherche qui ne considèrent que les aspects sociaux du milieu de vie sans prendre en compte les aspects biophysiques qui y sont associés ne sont pas à proprement parler des activités du domaine de l'ERE; inversement, une activité d'enseignement ou d'investigation qui ne s'intéresse qu'aux aspects biophysiques du milieu, en dehors de toute considération des relations qu'entretiennent les personnes et les groupes sociaux avec le milieu, demeure en périphérie de l'ERE.

7 En plus d'adopter une telle perspective environnementale complexe et systémique, une recherche en ERE adopte une perspective éducationnelle, c'est-à-dire qu'elle s'intéresse fondamentalement à des questions relatives au développement des personnes et des groupes sociaux en relation avec leur milieu de vie. Certes, l'importance relative de ces deux perspectives peut-être modulée, mais on y retrouve à la fois une préoccupation pour une (ou des) question(s) environnementale(s) et une (ou des) préoccupation(s) éducationnelle(s), d'ordre axiologique, curriculaire, pédagogique, didactique, politique, éthique ou autre ${ }^{2}$. À cet effet, on peut se demander par exemple, à quelles conditions une recherche portant sur les connaissances de base en écologie ou sur les résistances institutionnelles à la pédagogie de projet peut-elle se greffer au domaine de la recherche en ERE? Ou encore, comment une analyse de contenu de l'information en matière de politique environnementale dans les journaux peut-elle s'inscrire dans le champ de la recherche en ERE ? Enfin, quels aspects d'une recherche sur l'éducation au développement peuvent être mis en lien avec le domaine de la recherche en ERE?

8 Ces questions nous ont amenés à distinguer sommairement trois types de recherche au regard de leur rattachement au domaine spécifique de l'ERE (nous adaptons et illustrons ici la typologie esquissée par Santoire, 19993) :

9 Les recherches dont l'ERE est l'objet central s'intéresse à ses fondements, processus, résultats ou enjeux. Par exemple, les recherches (comme celle de Pruneau et Chouinard, 1997) qui visent à optimaliser chez les sujets le développement de compétences associées à la relation à l'environnement ou qui ont pour but la transformation des pratiques d'enseignement à cet effet (comme chez Daniel, $1997 \mathrm{ou}$ 
Goffin, 1997) ; de même, bien entendu, les recherches diagnostiques ou les bilans de la situation de l'ERE (telle l'étude de Cloutier, 1997).

D'autres recherches s'intéressent à l'ERE comme un aspect important, mais non central, de l'étude. Par exemple :

- l'ERE est un contexte où se déroule (où s'expérimente) un processus que l'on cherche à documenter (comme dans l'étude de Savoie-Zajc, 1997, sur le processus de recherche luimême comme stratégie de changement, ou celle de Langevin, 1997, sur l'animation pédagogique dans un contexte d'éducation à distance) ;

-1"ERE est envisagée comme un contexte privilégié où l'on pourra mettre à profit des compétences ou des stratégies qui ne lui sont pas spécifiques ou exclusives et sur lesquelles porte l'activité de recherche (comme dans la recherche de Tessier, 1997, sur l'analyse des éthiques en présence dans un dossier de presse) ;

- l'objet à l'étude n'est que l'une des caractéristiques ou conditions de l'ERE et cette caractéristique ou condition ne lui est pas exclusive (comme chez Wolf-Michael, 1997, qui traite de la désinstitutionnalisation des sciences comme facteur favorisant l'ERE) ;

- l'ERE n'est envisagée qu'en fonction d'une perspective spécifique, comme celle du développement durable (par exemple, chez Laurent, 1997 ou chez Vaillancourt, 1997).

Enfin, certaines recherches offrent des résultats ou des réflexions qui peuvent avoir une incidence sur l'ERE ou qui tiennent compte de l'éducation relative à l'environnement parmi d'autres préoccupations éducatives, mais leur lien de proximité avec l'ERE est plus ténu, parfois même marginal et non explicite. Ainsi en est-il des recherches sur les dimensions connexes de l'éducation contemporaine, comme l'éducation dans une perspective planétaire ou mondiale (Desroches, 1997; Ferrer, 1997 ; B. Gauthier, 1997) ou sur les représentations sociales dans les milieux d'intervention environnementale (comme chez Macchabée, 1997).

Deux observations s'imposent ici. D’une part, comme le mentionne Marie-Josée Santoire (1999), la démarcation n'est pas tranchée entre ces catégories (entre les recherches «au cœur » et "en périphérie » de l'ERE, et entre les recherches «en périphérie » et «satellites » de l'ERE). Une typologie est un outil d'analyse auquel les réalités ne se laissent pas aisément contraindre, surtout lorsqu'il s'agit d'objets complexes. Cette typologie méritera certes d'être affinée, peut-être transformée.

D'autre part, quel que soit leur lien de proximité avec le domaine spécifique de l'ERE, toutes ces recherches contribuent au développement de l'éducation relative à l'environnement et doivent être accueillies comme partie intégrante du " patrimoine » de recherche en ERE.

Enfin, il convient de distinguer les recherches du domaine de l'ERE de celles du domaine de la formation relative à l'environnement (FRE), et de prendre en compte leur degré d'interpénétration. Les travaux de Babacar (1997) et de Croizer (1997) s'inscrivent dans la catégorie de la recherche en FRE. Les éléments théoriques fournis par Goffin et Croizer, dans la section Regards de ce volume, aideront à préciser la distinction entre ces deux champs d'intervention et de recherche, souvent interreliés. 


\section{Qu'est-ce qui caractérise une activité de recherche en ERE ?}

Parmi les propositions de communication au colloque, nous avons reçu des descriptions de projets d'intervention ou des présentations d'outils pédagogiques récemment développés. Nous avons reçu également des textes réflexifs sur l'ERE (sur ses finalités par exemple). Ont alors émergé les questions suivantes : à quelles conditions un projet de développement et d'expérimentation d'un outil, d'un programme ou d'une stratégie devient-il aussi un projet de recherche (recherche-développement) ? Que faut-il ajouter à l'intervention (d'éducation ou de formation) pour qu'elle s'enrichisse d'une dimension de recherche (recherche-intervention, recherche-action, rechercheformation, recherche-évaluation ${ }^{4}$ ? La réflexion est-elle nécessairement une activité de recherche? Enfin, à quelles conditions un ensemble de réflexions spontanées, informelles et contextuellement situées peuvent-elles donner lieu à des éléments théoriques susceptibles de contribuer au développement de modèles explicatifs, praxéologiques, axiologiques ou autres ? La réponse à ces questions sera sans doute la même que pour le domaine de l'éducation en général (voire celui des sciences sociales), où les chercheurs reconnaissent de plus en plus le lien étroit entre l'intervention, la formation (ou développement professionnel), la réflexivité et la recherche (Schön, 1987 ; Hugon et Siebel, 1988 ; Saint-Arnaud, 1992 et autres).

Dans le cadre de l'organisation du colloque, nous avons adopté les balises suivantes:

- Une activité peut être considérée comme recherche 1) si son but est de développer de nouveaux savoirs, ou de consolider, d'approfondir ou de synthétiser des savoirs existants, et 2) si elle est menée avec rigueur et dans une perspective critique, ce qui implique pour le moins une distanciation entre le chercheur et son projet et de préférence, une confrontation avec d'autres façons de voir et de faire les choses.

- Une activité de recherche implique que ses acteurs clarifient et justifient les cadres théoriques et méthodologiques qu'ils adoptent ou proposent. Ces cadres sont parfois adoptés en amont des activités; parfois, ils sont progressivement construits en cours de projet. En réalité, nous savons que tout cela se passe souvent en rétroactions constantes.

- Quelle que soit la méthodologie adoptée, les chercheurs doivent faire preuve de transparence à cet effet, et ne pas hésiter à révéler s'il y a lieu des mouvements d'essais et d'erreurs, des incertitudes et des doutes, des écarts. Il y a donc d'abord un souci de rigueur et non pas d'autogratification.

- Une activité de recherche, lorsqu'elle est associée à l'intervention (et c'est souvent le cas en éducation), se caractérise par la réflexivité et la recherche d'éléments théoriques (descriptifs, explicatifs, stratégiques, méthodologiques ou critiques) transférables à d'autres situations. En somme, il s'agit de dépasser l'idiosyncrasie (singularité) des situations, tout en prenant en compte et même en valorisant le caractère singulier de chacune.

- Une activité de recherche implique un processus de validation des résultats : validation théorique (évaluation et discussion par des pairs ou des experts, ou encore des acteurs de la problématique étudiée) ou validation par l'expérimentation (au sens large).

- Un rapport de recherche gagne toujours également à se situer dans une "tradition », un courant d'histoire ou un patrimoine de recherche dans son domaine, à référer à d'autres auteurs, à d'autres processus et résultats, de façon à insérer son projet dans la construction d'une mosaïque globale des activités de recherche dans le domaine. 
17 De façon générale, se pose enfin la question de la scientificité. Qu'est-ce qu'une recherche scientifique? Toute recherche en éducation doit-elle être nécessairement qualifiée de scientifique? Peut-on considérer la recherche sans nécessairement se référer à la notion de science? Qu'en est-il par exemple d'une analyse conceptuelle s'inspirant de méthodes propres à la philosophie ? Dans la perspective épistémologique du constructivisme social, associé à la posture ontologique du relativisme critique, la recherche postmoderne en éducation remet en question les critères traditionnels de scientificité (Carr et Kemmis, 1986; Robottom et Hart, 1993; Constas, 1998 ; Sauvé, 1998). Dépouillée de ses attributs positivistes, la démarche « scientifique » n'est-elle pas réduite ici à répondre aux seuls critères de rigueur (associée à la distance critique) et de transparence méthodologique, pour la production de savoirs crédibles et transférables ou utiles? Ces questions, actuellement débattues dans les milieux de la recherche en éducation, concernent aussi bien entendu le domaine de la recherche en ERE. Pour le colloque, nous avons adopté une conception large de la recherche, répondant aux six critères ci-haut mentionnés et qui n'est pas restreinte à la conception traditionnelle (positiviste ou post-positiviste) de la scientificité.

\section{Quels types de recherche retrouve-t-on en ERE?} Kemmis (1986), ces derniers étant eux-mêmes inspirés de Habermas (1974). Robottom et Hart, dans leur excellent ouvrage intitulé Research in Environmental Education, ont identifié trois principaux types de recherche, qui se distinguent principalement par leurs postures ontologique, épistémologique et méthodologique : la recherche de type positiviste, de type interprétative, et de type critique. Nous présentons et adaptons (italique dans le texte) l'essentiel de cette typologie (déjà présentée dans Sauvé, 1997b) ; nous formulerons également quelques observations relatives aux productions et propositions de recherche sommairement analysées dans le cadre de ce colloque.

La recherche de type positiviste s'appuie sur une ontologie réaliste (les objets ont une existence propre, en dehors du sujet qui les appréhende) et sur une épistémologie objectiviste (la démarche scientifique empiriste, si elle est rigoureusement "suivie " permet au sujet de "découvrir " l'objet dans sa réalité propre). Elle vise surtout à expliquer, à généraliser, à prédire. Elle adopte à cet effet une méthodologie expérimentale et des stratégies quantitatives. Une telle recherche est menée par des experts externes, qui suivent rigoureusement le design de recherche qu'ils ont établi a priori. La recherche de type post-positiviste adopte plusieurs de ces prémisses, mais elle reconnaît l'influence des schèmes de perception du sujet sur la façon dont ce dernier appréhende 
l'objet (ontologie réaliste critique) et ses propositions méthodologiques sont par ailleurs plus diversifiées.

21 Robottom et Hart (1995), de même que Wals (1990) déplorent que la recherche en éducation relative à l'environnement ait été jusqu'ici trop restreinte au type positiviste ou post-positiviste et qu'elle ait été surtout associée à une intervention de type behavioriste. Tout le courant de la recherche sur les variables explicatives du "comportement civique responsable» (ensemble de recherches synthétisées par Hungerford et Volk, 1990) se rattache à ce type de recherche. Il s'agit d'isoler des variables (comme la connaissance des concepts écologiques de base) et de vérifier leur relation de cause à effet sur le comportement à l'égard de l'environnement, en utilisant par exemple des questionnaires fermés avec échelles de type Likert.

22 La recherche de type interprétative s'appuie sur une ontologie relativiste (la réalité n'existe qu'en fonction du schème mental à travers lequel elle est appréhendée) et sur une épistémologie subjectiviste (le sujet construit l'objet; n'est considérée que cette représentation dont on reconnaît par ailleurs que de nombreux éléments - sinon tous les éléments - sont socialement construits). Ce type de recherche s'intéresse aux significations de la réalité pour ceux qui y sont associés: elle a une visée herméneutique et accorde une grande importance au contexte. Elle adopte des stratégies méthodologiques qualitatives. Elle est menée par des experts externes, qui conçoivent un design de recherche souple, adaptable.

La recherche de type interprétative est plutôt récente en éducation relative à l'environnement, comme le signale Robertson (1994), et elle reste à développer. À titre d'exemple, citons la recherche de Sauvé et Garnier (1999) concernant l'élaboration d'une phénoménographie des représentations de l'environnement auprès de diverses populations.

La recherche de type critique s'appuie sur une ontologie qualifiée de réaliste critique (les objets ont une existence réelle, mais ils ont différentes significations selon le champ symbolique au sein duquel ils sont appréhendés) et sur une épistémologie intersubjectiviste et dialectique (le savoir est issu d'un réseau d'interactions sujet - sujets - objet ; il est socialement construit et il est déterminé par le contexte historique, social, éthique, etc. où il s'élabore). Ce type de recherche est orienté vers l'action ; il s'agit de produire un savoir critique de nature à catalyser le changement social. La recherche critique est essentiellement participative ; elle est cogérée par les différents acteurs de la problématique abordée et elle se préoccupe de développer le pouvoir-faire de ces derniers. Elle adopte une multiméthodologie, privilégiant les stratégies qualitatives, mais exploitant également au besoin des outils quantitatifs. Son design est négocié entre les partenaires et essentiellement adaptatif, voire émergent au fil de la démarche.

À titre d'exemples de questionnement critique en éducation relative à l'environnement, Robottom et Hart (1993) proposent entre autres: Quels sont les intérêts servis par les choix relatifs aux politiques et aux pratiques de l'ERE à l'école? Comment le savoir relatif à l'environnement est-il produit et reproduit à l'école ? On pourrait également se demander: les structures institutionnelles de l'école actuelle entravent-elles la mise en œuvre d'une pédagogie adaptée à l'ERE? quel processus de changement peut-on envisager à cet effet ? La recherche-action (et autres formes de recherche-intervention), la recherche-évaluation, la recherche-développement peuvent être menées dans une approche critique. John Elliott (1994) et Michela Mayer (1997) témoignent en particulier de la fécondité de la recherche-action comme processus de changement en éducation, et plus spécifiquement en éducation relative à 
l'environnement. Pourtant, comme le signalent Robottom et Hart (1993), la recherche critique émerge à peine en ERE.

Ainsi, du point de vue de la finalité, la recherche de type positiviste est davantage explicative et prédictive (concernant la «découverte» de la réalité et des lois inhérentes, en particulier celles qui régissent les relations de cause à effet), la recherche de type interprétative est herméneutique (favorisant la mise au jour des significations) et la recherche de type critique est essentiellement stratégique (visant à stimuler la production collective de savoirs de type critique dans le but de favoriser la pertinence contextuelle de l'action éducative en vue de changements sociaux).

Ces trois types de recherche sont mutuellement exclusifs quant à la conjugaison de leurs postures ontologique, épistémologique et méthodologique; ils peuvent offrir toutefois trois regards de recherche complémentaires lorsqu'il s'agit d'étudier un objet complexe (comme dans le cas d'une étude diagnostic de la situation de l'ERE dans un milieu). Habermas (1974) invite d'ailleurs à l'exploitation complémentaire de ces trois perspectives paradigmatiques, à condition de reconnaitre leurs fondements respectifs et les implications de ces derniers.

Enfin, il importerait sans doute de revoir cette typologie et les jugements de valeurs qui sont associés aux différents types de recherche en ERE. Les observations (fondées ou non) de Connell (1997) sur les méprises et les critiques injustifiées à l'égard de la méthodologie empirique-analytique en ERE, de même que celles de Walker (1997) sur les limites de la recherche critique en ERE pourraient contribuer à la discussion sur le sujet.

Quoi qu'il en soit, l'exploration sommaire des productions de recherche que nous avons repérées au sein de la francophonie $(\mathrm{n}=84)^{5}$ et l'analyse des propositions de communication au colloque $(n=56)$ nous permettent de constater qu'il y a peu de recherches adoptant prioritairement des stratégies de type positiviste (comme l'étude de Belhareth, 1997). Ces dernières sont surtout utilisées pour la caractérisation des contextes et pour le traitement de certaines données de diagnostic ou de bilan (par exemple, Grodya, 1997). La recherche francophone en ERE, fort peu publiée jusqu'ici, apparaît plus récente que la recherche anglophone, et semble avoir échappé au courant positiviste - behavioriste qui a marqué les productions anglo-saxonnes des années '70 et '80 (comme en témoigne le Journal of Environmental Education). La recherche francophone, quand elle parvient à se structurer, à dépasser la simple description de projets ou le rapport d'évaluation, et qu'elle se penche sur la théorisation de l'action, rejoint davantage les nouvelles tendances réflexives et critiques qui apparaissent clairement dans les revues spécialisées plus récentes, comme le Canadian Journal of Environmental Education ou Environmental Education Research, et qui ont caractérisé jusqu'ici les productions du Australian Journal of Environmental Education.

30 Mais s'il est une caractéristique de la recherche francophone en ERE, c'est l'intérêt porté à la recherche de type interprétative. Plusieurs études se penchent sur les conceptions et les représentations liées à l'environnement ou encore sur la pratique de l'ERE chez les intervenants (comme chez El Hattab, 1997); ces recherches adoptent une approche phénoménologique et une méthodologie de type qualitative (par exemple, Dionne, 1997 et A. Roy, 1997). Le champ théorique des représentations sociales inspire également plusieurs recherches récentes (par exemple, Garnier, 1997, Ponton, 1997 et Picard, 1995). Les méthodologies d'inspiration ethnographique, comme celle de Dubé (1997), permettent également de pénétrer l'univers des significations. Enfin, l'apport 
des sciences de la communication à la dynamique des projets en ERE intéresse désormais certains chercheurs (à titre d'exemple, l'étude de Fauteux, 1997). La recherche de type critique n'est que rarement identifiée comme telle par les auteurs; le cadre de référence de la théorie critique (synthétisé entre autres dans Sauvé, 1997 c) semble peu connu des chercheurs de la francophonie. Mais de nombreuses productions de recherche s'inscrivent toutefois dans une visée critique (au sens large), de type réflexive (comme celles de Berryman, 1997 ; de Desautels, 1997 ; de Guilbert, 1997 ; de Bader, 1998), de type analytique (Pelletier et Lefebvre, 1997) ou de type transformative (par exemple, B. Gauthier, 1997 ; Pruneau et Chouinard, 1997 ; Savoie-Zajc, 1997). Dans ce dernier cas, la recherche-action trouve une place privilégiée (comme en témoignent entre autres, Poudrier, 1996; Sow, 1996; Lalonde, 1997). La caractérisation des recherches à l'aide de la typologie tripolaire présentée ci-haut n'est pas sans poser problème.

- D'une part, de nombreuses recherches adoptent différentes postures, à différents moments de l'investigation, ou encore, intègrent en les recadrant des choix qui pourraient être divergents. Rooney (1997) montre par exemple la contribution potentielle importante de la recherche sur les représentations sociales (de type interprétative), à la résolution de la crise environnementale contemporaine (visée critique), que la chercheure invite à interpréter comme une construction sociale. Ainsi, le projet de recherche de Ponton (1998), qui porte sur les représentations et pratiques urbaines relatives à l'environnement chez les adolescents d'un quartier défavorisé de Montréal, dépasse la perspective interprétative pour entrer dans une démarche de critique sociale avec les sujets eux-mêmes, en vue de la transformation des réalités qu'ils perçoivent comme problématiques. De même, la recherche de Dubé (1997) dépasse la mise au jour des significations et s'inscrit dans le courant de l'ethnographie critique, qui engage les acteurs de la situation problématique dans une démarche d'exploration de leur propre univers symbolique en vue d'améliorer leur réalité environnementale, sociale et éducationnelle.

- D'autre part, cette typologie tripolaire apparait réductrice à l'égard de la diversité des possibilités de choix de recherche. Certains chercheurs (par exemple, B. Gauthier, 1997 ; Guilbert, 1997 et L. Roy, 1997, à la suite de Janse van Rensburg, 1994) préfèrent se rattacher à ce qu'ils appellent le courant réflexif en ERE, qui n'adopte pas nécessairement toutes les prémisses de la recherche de type critique surtout en ce qui concerne les propositions néomarxistes également, le courant de la recherche féministe en ERE (analysé par Gough, 1997) vise la transformation des réalités, mais se sent à l'étroit dans l'approche exclusivement rationnelle du courant critique.

- Enfin, plusieurs recherches sont difficiles à classer selon cette typologie axée sur les courants paradigmatiques de recherche les plus souvent considérés; en réalité, les pratiques de recherche sont plurielles, mouvantes, adaptatives: elles peuvent difficilement être enfermées dans un catalogue à trois entrées. Par exemple, certaines recherches visent le changement pour l'amélioration des pratiques en cours et adoptent à cet effet une approche collaborative, mais elles ne s'inscrivent pas pour autant dans une démarche de critique sociale et éducationnelle fondamentale.

31 Sans nier d'aucune façon l'intérêt et la pertinence de la typologie précédente, il nous est apparu toutefois intéressant de développer d'autres outils de classification: la multi-typologie suivante (développée en collaboration avec Santoire, 1999) permet de porter d'autres regards sur différentes facettes des recherches en ERE. Il ne s'agit certes pas d'un outil exhaustif, mais il apparaît simple et utile dans la perspective d'une première exploration. 

évaluation, la recherche interprétative et la recherche-intervention (dont la rechercheaction). À noter que certaines recherches, poursuivant plus d'un but, peuvent correspondre à plus d'un type à la fois. Soulignons enfin que l'importance de la recherche-évaluation est particulièrement mise en évidence dans les travaux du réseau européen REVERE - Recherche sur l'évaluation en éducation à l'environnement - dirigé par Louis Goffin. En témoigne entre autres la recherche de Taelman (1997), présentée au colloque.

\begin{tabular}{|c|c|c|}
\hline TYPES DE RECHERCHE & VISÉE OU BUT & $\begin{array}{l}\text { IMPORTANCE } \\
\text { RELATIVE }\end{array}$ \\
\hline Recherche théorique & $\begin{array}{l}\text { Développer des éléments théoriques: } \\
\text { concepts, modèles, typologie, etc. }\end{array}$ & $7 \%$ \\
\hline Recherche descriptive & $\begin{array}{l}\text { Décrire un objet ou un phénomène, } \\
\text { le caractériser. }\end{array}$ & $14 \%$ \\
\hline $\begin{array}{l}\text { Recherche expérimentale } \\
\text { (ou plus justement, quasi-expérimentale) }\end{array}$ & $\begin{array}{l}\text { Éablir des liens de cause à effet en } \\
\text { manipulant au moins une variable } \\
\text { indépendante et en observant ses effets } \\
\text { sur une ou des variables dépendantes. } \\
\text { Idéalement, les sujets étudiés sont } \\
\text { choisis aléatoirement. }\end{array}$ & nil \\
\hline Recherche interprétative & $\begin{array}{l}\text { Mettre au jour la signification des réalités } \\
\text { chez les sujets ou acteurs d'une situation ; } \\
\text { étudier leurs représentations ou plus } \\
\text { spécifiquement leurs conceptions, } \\
\text { leurs attitudes, leurs valeurs, etc. }\end{array}$ & $25 \%$ \\
\hline $\begin{array}{l}\text { Recherche-intervention } \\
\text { - recherche-action } \\
\text { - recherche-formation } \\
\text { - recherche pour l'innovation }\end{array}$ & $\begin{array}{l}\text { Induire et documenter un changement. } \\
\text { Dans le cas de la recherche-action, associer } \\
\text { action et réflexion pour faire émerger } \\
\text { une théorie de l'action. }\end{array}$ & $25 \%$ \\
\hline Recherche-développement & $\begin{array}{l}\text { Développer de nouveaux objets } \\
\text { (théoriques ou concrets) ou } \\
\text { de nouveaux procédés. }\end{array}$ & $19 \%$ \\
\hline $\begin{array}{l}\text { Recherche-évaluation : } \\
\text { - recherche évaluative } \\
\text { - recherche diagnostique } \\
\text { - autres recherches lices à l'évaluation }\end{array}$ & $\begin{array}{l}\text { Déterminer la pertinence, la désirabilité, } \\
\text { la qualité ou autres paramètres d'un objet } \\
\text { (théorique ou concret). Développer des } \\
\text { savoirs théoriques et stratégiques sur } \\
\text { l'ćvaluation elle-même. }\end{array}$ & $28 \%$ \\
\hline
\end{tabular}

34 Outre leur(s) visée(s), il est également possible de caractériser les recherches en fonction de leur approche méthodologique dominante. À cet effet, on peut identifier trois catégories (en collaboration avec Santoire, 1999) :

- L'approche qualitative : cette approche implique une collecte de données essentiellement qualitatives (surtout narratives) lors d'une étude qui prend en compte plusieurs variables interreliées, compte tenu de la complexité de l'objet et du contexte, et sur lesquelles il n'y a ni intervention, ni contrôle. L'approche qualitative n'a pas recours à des catégories d'analyse prédéterminées : les catégories émergent par induction, au fil de l'analyse des données. Les hypothèses se structurent et évoluent en fonction de l'observation progressive des réalités. 
- L'approche quantitative : cette approche implique la collecte de données numériques en fonction de catégories de recherche déterminées a priori. L'étude porte sur une ou quelques variables sur lesquelles il y a ou non manipulation (selon qu'il s'agit d'une recherche expérimentale ou d'une recherche descriptive, liée à l'observation). Les hypothèses sont le plus souvent posées a priori et sont vérifiées par l'analyse des données.

- L'approche hybride : cette approche fait intervenir à divers degrés et à différents moments de la recherche, certaines stratégies caractéristiques des deux approches.

L'analyse de contenu de l'ensemble des résumés de recherche en ERE (sur les fiches ou dans les documents consultés) montre une nette prévalence de l'approche qualitative. Un troisième outil typologique propose une classification des recherches selon l'interaction entre le ou les chercheur(s) et les acteurs de la situation étudiée. Trois types de recherche sont envisagés (en collaboration avec Santoire, 1999):

- La recherche exogène, qui est menée par un chercheur ou un groupe de chercheurs sans faire appel aux acteurs de la situation étudiée ;

- La recherche collaborative, qui, en totalité ou en partie, fait appel à un ou des acteurs de la situation étudiée pour l'atteinte d'objectifs définis par le chercheur (ou l'équipe de recherche).

- La recherche participative, qui fait appel à la participation des acteurs de la situation étudiée pour l'ensemble des décisions et des activités de recherche. Certes, de façon réaliste, il faudrait reconnaître ici l'existence d'un gradient de participation et différents créneaux où cette dernière peut s'exercer.

Les données recueillies par Santoire (1999) permettent d'observer que les chercheurs n'explicitent pas a priori cette dimension d'interaction entre les acteurs de la recherche. Selon les informations disponibles (73 fiches et résumés de documents), $11 \%$ des recherches seraient associées à la recherche de type collaborative ou participative. Il faudrait avoir accès aux documents de recherche eux-mêmes pour vérifier ces résultats provisoires. Enfin, un dernier critère de classification est intégré à notre multitypologie de recherche : celle du degré d'approfondissement du sujet. Ici intervient la notion de recherche exploratoire, qui apparaît appropriée lorsque l'objet est encore inexploré et que l'étude vise à en faire ressortir, dans un premier temps, les éléments les plus caractéristiques ou l'essentiel de la problématique associée, et à identifier des hypothèses à vérifier ou des pistes de recherche à poursuivre. Selon les données de Santoire (1999), 7 \% des fiches du répertoire (Québec et Est du Canada) ont signalé des recherches de type exploratoire.

Il importe de souligner le caractère très approximatif de ces résultats qui émanent d'une première exploration auprès d'une population spécifique de chercheurs (ceux du Québec et de l'Est du Canada) et à partir de sources secondaires (fiches et résumés extraits des documents consultés). D’ailleurs, ce ne sont pas les résultats quantitatifs obtenus qui présentent un intérêt ici, mais le fait que cet exercice d'analyse ait été associé à l'élaboration d'outils typologiques simples et complémentaires pour faciliter d'éventuelles recherches qui dépasseront le stade exploratoire. 


\section{Quels sont les objets privilégiés de la recherche en ERE?}

Le tableau 2 présente un système de classification de la recherche en ERE en fonction des objets de recherche. Ces catégories ont émergé de l'analyse des données fournies sur les fiches par les auteurs eux-mêmes (du Québec et de l'Est du Canada) ou de l'analyse des résumés de recherche obtenus en bibliothèque (Santoire, 1999). On peut observer que trois champs de recherche sont particulièrement exploités chez les chercheurs de la francophonie nord-américaine : les aspects relatifs à l'enseignement et l'apprentissage au sein de la situation pédagogique, les aspects liés à la subjectivité de l'apprenant (attitudes, valeurs, représentations) et enfin, la formation des formateurs.

Ce dernier champ tente de contribuer à résoudre l'un des principaux facteurs limitants du développement de l'ERE (Sauvé et coll., 1997) : à cet effet, signalons en particulier les recherches de Bruxelles (1997) et de Souchon et Raichvarg (1997) présentées au colloque. La recherche de Bruxelles s'inscrit dans le cadre des travaux de l'Ifrée (Institut de recherche et de formation en éducation à l'environnement, France) qui associe précisément recherche et formation.

Les catégories du tableau 2 fournissent un premier aperçu des objets de recherche privilégiés en ERE. Les catégories proposées ne sont toutefois pas exhaustives et la typologie devra être affinée. Santoire souligne en effet les limites de sa recherche de type exploratoire, dont l'un des buts est de fournir des pistes et des outils pour poursuivre et parfaire le travail d'analyse. Ainsi, il serait certes intéressant de mettre en relation les objets de recherche et les types de recherche correspondants ; le choix des objets est en effet étroitement lié aux choix paradigmatiques et stratégiques de recherche. Malheureusement, dans le cadre de cette première recherche exploratoire, les données disponibles ne permettent pas une telle analyse.

Tableau 2 : Répartition des recherches selon les objets (Santoire, 1999)

\begin{tabular}{ll}
\hline OBJETS DE RECHERCHE & $\begin{array}{l}\text { Fréquence } \\
\mathrm{n}=73\end{array}$ \\
\hline $\begin{array}{l}\text { Pédagogie, didactique et curriculum } \\
\text { (stratégies d'apprentissage, modèles } \\
\text { pédagogiques, design pédagogique) }\end{array}$ & 34 \\
\hline Attitudes et valeurs & 14 \\
\hline Représentations (sociales) & 12 \\
\hline Formation de formateurs en ERE & 11 \\
\hline Communication, médias et muséologie & 5 \\
\hline Fondements de l'ERE, philosophie, sociologie et éthique & 5 \\
\hline État de la situation, bilan, diagnostic & 3 \\
\hline $\begin{array}{l}\text { Dimensions éducationnelles associées à l'ERE : } \\
\text { éducation globale, dans une perspective planétaire, } \\
\text { pour un développement durable, etc. }\end{array}$ & 2 \\
\hline
\end{tabular}




\section{Quelle est la pertinence éducationnelle et sociale de la recherche actuelle en ERE ? Comment renforcer cette double pertinence?}

41 Au moment de se pencher sur le bilan, les enjeux et les perspectives de la recherche en éducation relative à l'environnement, s'est également posée la question fondamentale de la pertinence éducationnelle et sociale de la recherche en ERE. À quoi et à qui sert la recherche en ERE? À quoi et à qui devrait-elle optimalement servir? Par qui cette recherche est-elle actuellement menée ? Qui devrait-elle interpeller?

À défaut de pouvoir traiter adéquatement chacune de ces questions, nous nous attarderons davantage à la pertinence éducationnelle de la recherche en ERE, en exploitant une troisième typologie de la recherche (Sauvé, 1997 b), inspirée entre autres des propositions de classification de Carr et Kemmis (1986), De Landsheere (1992), Fontaine (1994) et Van der Maren (1995). Cette typologie identifie trois catégories de recherche, caractérisées par le but et la perspective (posture) adoptés : la recherche au sujet de l'éducation, en éducation, pour l'éducation.

43 La recherche au sujet de (ou sur) l'éducation aborde les réalités éducatives de l'extérieur, pour les décrire, les expliquer, les prédire, «les mettre en relation avec d'autres grandes variables sociales, économiques, organisationnelles et politiques » (Fontaine, 1994). Elle utilise fréquemment (mais non exclusivement) les stratégies caractéristiques de la recherche positiviste. En éducation relative à l'environnement, on pourra tenter d'identifier par exemple les conditions institutionnelles qui prévalent dans les milieux scolaires où l'ERE est intégrée; ou encore, on s'intéressera aux caractéristiques socioculturelles des enseignants qui intègrent l'ERE à leur pratique éducative. Le bilan de Dubé et Orellana (1997), comme le diagnostic de Acosta (1999), incluent par exemple l'identification ou la caractérisation des acteurs et des initiatives en ERE.

La recherche en éducation rejoint deux préoccupations différentes. D'une part, elle se préoccupe de comprendre les réalités associées aux situations éducatives, en y portant un regard "de l'intérieur", comme dans la recherche de type phénoménologique (Picard, 1995) ou ethnographique (Dubé, 1997); il s'agit alors d'une recherche dans l'éducation, de type interprétatif, préoccupée surtout d'explorer les significations des réalités pour les acteurs qui y sont associés. En ERE, on pourra trouver par exemple une étude de la diversité des représentations de l'éducation relative à l'environnement chez les enseignants et de l'impact de ces représentations sur les pratiques (DeMelo, 1997, Sauvé et Bégin, 1997). D'autre part (selon Fontaine, 1994), la recherche en éducation vise à améliorer les pratiques éducatives (mais sans nécessairement adopter une visée de transformation). La recherche quasi-expérimentale, certains types de rechercheaction (liée par exemple à l'essai d'innovations) et la recherche évaluative sont parmi les types de recherche qui peuvent alors être exploitées. En ERE, on pourra par exemple expérimenter un modèle pédagogique particulier dans un contexte spécifique, de façon à en vérifier la pertinence (la validité écologique) et à y apporter s'il y a lieu, des modifications ou des améliorations (Sow, 1996). Notons que la recherche en éducation, comme la recherche au sujet de l'éducation, peut être menée dans une perspective 
critique, et présenter de ce fait certaines caractéristiques d'une recherche pour l'éducation, telle que définie ci-après.

La recherche pour l'éducation vise essentiellement le développement de l'éducation. Trois conceptions se retrouvent ici :

- Une recherche pour l'éducation se distingue d'une recherche au sujet de l'éducation, en ce qu'elle est essentiellement critique et vise à transformer les réalités éducatives (Carr et Kemmis, 1986, p. 156): elle est axée sur le changement; elle suppose une approche participative et collaborative; elle est arrimée à une expérience éducative concrète; elle s'appuie sur un processus de recherche-action intégrale (Morin, 1992), au cœur de laquelle se retrouve l'exercice rigoureux d'une véritable praxis, soit une théorisation critique des actions éducatives habituelles (selon la définition de Van der Maren, 1995, p. 50).

- Mais selon Van der Maren (1995), une recherche pour l'éducation ne doit pas se limiter à la praxis : elle doit intégrer à la fois les savoirs issus de la praxis (c'est-à-dire de la réflexion critique sur l'action, qui fournit des signaux perceptibles pouvant être répertoriés et paramétrisés) et les savoirs appliqués (qui opérationnalisent les résultats de la science en suivant une démarche de résolution de problèmes), de façon à générer un savoir pour l'action, appelé savoir stratégique.

- Enfin, selon Fontaine (1994), une recherche pour l'éducation doit intégrer à la fois la recherche au sujet de et la recherche en éducation ; ces deux approches s'interfécondent en effet, pour induire une dynamique d'amélioration des politiques et des pratiques éducatives.

Tous les auteurs s'accordent à déplorer qu'une recherche pour l'éducation reste encore à développer. Or le domaine de l'éducation relative à l'environnement apparaît particulièrement fécond à cet effet, puisqu'il s'agit de transformer les réalités éducationnelles en vue de favoriser les transformations sociales et environnementales qui s'imposent pour résoudre les problèmes liés au réseau de nos relations avec le milieu de vie. La recherche de Calderfet, puisqu'il s'agit de transformer les réalités éducationnelles en vue de favoriser les transformations sociales et optimaliser son implantation, jugée favorable au changement social. Orellana (1997) analyse la dynamique des communautés d'apprentissage en ERE en vue de favoriser des processus d'apprentissage plus pertinents, impliquant l'ouverture de l'école sur la communauté, dans la perspective de l'avènement d'une société éducative. Enfin, Marquis (1997) et Toussaint et coll. (1997) abordent un champ de recherche de plus en plus exploré, celui du lien entre l'ERE et les questions de développement.

De façon générale, la pertinence éducationnelle et sociale de la recherche en ERE (ou pour le moins la préoccupation de changement) transparaît dans les intentions des recherches en cours. Non seulement retrouve-t-on de nombreuses recherches axées directement sur le changement (éducationnel, social, environnemental), mais on observe que les recherches au sujet de et dans l'éducation s'inscrivent la plupart du temps dans de plus vastes projets de transformation. Cette double pertinence peut toutefois être accentuée si les conditions d'une véritable recherche critique, associée à une recherche pour l'éducation, sont mises en œuvre (Carr et Kemmis, 1986 ; Hart, 1991, 1993 ; Robottom et Hart, 1993 ; Chevrier, 1994 ; Collectif, 1995 ; Van der Maren, 1995 : synthèse présentée Dans Sauvé, 1997 b) :

- Il importe d'adopter une approche globale et systémique des réalités, seule capable de prendre en compte la complexité des objets d'étude. Or l'objet de l'éducation relative à l'environnement (soit le réseau des relations personne - groupe social - environnement) est d'une extrême complexité. Le plus souvent, il faut recourir à une approche multi- 
méthodologique, qui permet d'associer en complémentarité diverses approches et stratégies de recherche.

- Une posture critique s'impose également, de façon à favoriser le repérage et la transformation des réalités qui posent problème. En ERE, il s'agit de transformer simultanément des réalités éducationnelles, sociales et environnementales, étroitement reliées les unes aux autres.

- Chaque situation éducative est unique, éminemment contextuelle. La recherche doit tenir compte de cette contextualité et doit même la valoriser plutôt que de chercher à l'occulter de façon à pouvoir généraliser. On se préoccupera plutôt, s'il y a lieu, du transfert des résultats à d'autres contextes, où les propositions pourront être adaptées ou modifiées.

- La recherche ne doit plus être l'apanage exclusif des chercheurs universitaires. Elle ne doit pas s'enfermer dans des discours hermétiques et des pratiques figées. Elle doit s'intéresser aux réalités éducatives concrètes, préoccupantes. Elle doit susciter la participation des divers acteurs de l'action éducative, de façon à ce qu'ils s'approprient véritablement cette recherche pour l'éducation. À cet effet, la recherche de type collaborative et participative, menée en co-gestion entre les partenaires, est particulièrement appropriée.

- La recherche pour l'éducation doit être arrimée à la pratique éducative et associée à une démarche de résolution de problèmes réels. Elle se préoccupe d'être pertinente et utile.

- Une recherche ainsi associée à l'action éducative offre un contexte privilégié de développement professionnel pour ses différents acteurs. Elle peut devenir entre autres le creuset du développement d'une pratique réflexive (Schön, 1987). Hugon et Siebel (1988) sont parmi les auteurs qui proposent le concept de recherche-formation.

- La recherche de type critique ne peut être véritablement achevée si elle n'est pas diffusée, de façon à être confrontée et de façon également à favoriser le transfert des résultats pour stimuler ou appuyer de nouvelles démarches de résolution de problèmes. Tous les acteurs d'une telle recherche devraient être invités au processus de diffusion qui s'ensuit: le discours et la « parole » de recherche doivent être partagés.

Lors des discussions qui ont eu lieu au cours du Colloque international de Montréal, différents participants ont signalé la pertinence et la désirabilité de l'une ou l'autre de ces conditions. Il importera de promouvoir un travail d'appropriation de ce type d'investigation par les acteurs du domaine de l'éducation. Cette tâche n'est certes pas aisée : cela implique de recourir à des approches et à des stratégies différentes, qui bousculent les traditions et qui restent à explorer, à parfaire, à opérationnaliser.

Par ailleurs, la reconnaissance de la pertinence d'une recherche critique en ERE s'inscrit dans une double problématique. D'une part, il s'agit d'une recherche engagée (comme la recherche sociale en général) : elle vise le développement de l'éducation relative à l'environnement, considérée comme une dimension fondamentale de l'éducation contemporaine et un facteur de changement socio-environnemental. Or une telle recherche (engagée) peut être perçue comme suspecte au regard des exigences d'une recherche dite "pure ", qui suppose une nécessaire distanciation de son objet d'étude. D'autre part, la recherche en ERE est également perçue comme suspecte dans les milieux d'intervention : en particulier, il subsiste de forts préjugés à l'égard de la recherche universitaire ou institutionnelle. Cette dernière semble peu crédible ou menaçante auprès des gens de terrain, même si elle est menée dans et avec le milieu, en partenariat, et si elle donne la parole aux acteurs mêmes du milieu. On dit volontiers que la recherche universitaire est concoctée dans des tours d'ivoire et qu'elle porte un regard critique qui ne sait pas tenir compte des contraintes du milieu. Un 
dialogue reste certes à établir et une culture du partenariat de recherche doit être développée.

Mais surtout, il faut reconnaître que la recherche pour l'éducation, associée au courant critique, plus encore que tout autre type de recherche, ne peut pas être ponctuelle, momentanée, discrète (au sens mathématique). Elle n'est pas linéaire, elle ne s'arrête pas aux premiers résultats, elle ne règle pas les problèmes une fois pour toutes. Elle est itérative et doit être envisagée comme un mode de vie constant au sein des milieux d'intervention éducative, comme une pratique en évolution, comme un incessant processus d'investigation critique sur elle-même.

\section{Conclusion}

51 Le Colloque international sur la recherche en ERE a donc été l'occasion non seulement de présenter les résultats de recherches récemment terminées ou la démarche de projets en cours, mais il a suscité une réflexion sur les activités de recherche ellesmêmes. Ont émergé des questions relatives à la spécificité de la recherche en ERE (et en particulier de la recherche francophone, peu connue et rarement discutée jusqu'ici), aux caractéristiques d'une telle recherche, à sa pertinence et à ses voies de développement privilégiées. Cet article présente certains éléments de réponse, dans le but de susciter la discussion et la poursuite d'une plus vaste démarche d'investigation sur le sujet.

52 Cette première exploration montre que la recherche francophone en ERE, bien que peu soutenue et diffusée, est déjà relativement riche et diversifiée. Elle semble avoir la vigueur de sa jeunesse. Elle apparaît créative, à l'affût de courants novateurs. Les acteurs de l'ERE découvrent progressivement son rôle fondamental pour enrichir l'intervention d'une dimension réflexive, soutenir une action éducative pertinente et contribuer à donner une plus grande crédibilité à cette dimension fondamentale de l'éducation contemporaine qu'est l'éducation relative à l'environnement.

Enfin, il y aurait avantage à développer de nouveaux projets de recherche visant à poursuivre l'analyse des productions actuelles, mais cette fois plus en profondeur et avec des outils conceptuels et typologiques appropriés. Le travail d'exploration mené en collaboration avec Santoire (1999) fournit des pistes qui pourraient être mises à profit à cet effet. En particulier, il serait intéressant de faire le bilan des résultats des recherches menées jusqu'ici : globalement, que proposent-elles, que suggèrent-elles ou qu'observent-elles en ce qui a trait à la théorie et à la pratique de l'ERE? Et que nous apprennent-elles sur les enjeux et la méthodologie de la recherche en ERE?

\section{BIBLIOGRAPHIE}

Acosta, S.P. (1999). Éléments théoriques et stratégiques pour l'intégration de l'éducation et de la formation relatives à l'environnement en milieu universitaire - Adaptation au contexte de l'Université 
Autonome Gabriel René Moreno, Santa Cruz de la Sierra, Bolivie. Mémoire de maîtrise en éducation, Université du Québec à Montréal.

Babacar, N.D. (1997). Former le monde rural à l'environnement. Colloque international - La recherche en éducation relative à l'environnement : bilan, enjeux et perspectives. 5 et 6 novembre 1997, Université du Québec à Montréal, Montréal.

Bader, B. (1998). Une vision socialisée des sciences au service d'une éducation relative à l'environnement socioconstructiviste. Canadian Journal of Environmental Education, 3, 156-170.

Belhareth, T. (1997). Application de la méthode statistique à l'inventaire de l'ERE dans le système éducatif tunisien. Colloque international - La recherche en éducation relative à l'environnement : bilan, enjeux et perspectives. 5 et 6 novembre 1997, Université du Québec à Montréal, Montréal.

Berryman, T. (1997). Littérature et environnement : explorer et décrire des relations afin d'y œuvrer. Colloque international - La recherche en éducation relative à l'environnement : bilan, enjeux et perspectives. 5 et 6 novembre 1997, Université du Québec à Montréal, Montréal.

Bruxelles, Y. (1997). Être partenaires pour former à l'écocitoyenneté des formateurs d'enseignants. Colloque international - La recherche en éducation relative à l'environnement : bilan, enjeux et perspectives. 5 et 6 novembre 1997, Université du Québec à Montréal, Montréal.

Calderón, N. (1997). Problématiques de l'application de la loi nationale colombienne en ERE. Colloque international - La recherche en éducation relative à l'environnement : bilan, enjeux et perspectives. 5 et 6 novembre 1997, Université du Québec à Montréal, Montréal.

Carr, W. et Kemmis, S. (1986). Becoming critical. Geelong : Deakin University Press.

Chevrier, J. (1994). La recherche en éducation. Montréal : Les Éditions Logiques.

Cloutier, C. (1997). Quelques résultats de l'état de la situation et des perspectives d'avenir de l'ERE des pays ayant le français en partage. Colloque international - La recherche en éducation relative à l'environnement : bilan, enjeux et perspectives. 5 et 6 novembre 1997, Université du Québec à Montréal, Montréal.

Collectif. (1995). Changement sociétal et recherche en éducation. Actes du Colloque, août 1994, Université du Québec à Chicoutimi, dans le cadre du programme de doctorat de l'Université du Québec. Chicoutimi : Les Éditions ERE-2000.

Connell, S. (1997). Empirical-analytical methodological research in environmental education : response to a negative trend in methodological and ideological discussion. Environmental Education Research, 3(2), 117-132.

Constas, M.A. (1998). Deciphering postmodern educational research. Educational Researcher, 27(9), 36-41.

Croizer, C. (1997). L'évaluation : instrument d'amélioration permanente des formations postgraduées en environnement. Colloque international - La recherche en éducation relative à l'environnement : bilan, enjeux et perspectives. 5 et 6 novembre 1997, Université du Québec à Montréal, Montréal.

Daniel, R. (1997). Formation des enseignants à l'ERE et changement des pratiques éducatives : une étude du cas de l'IUFM à Toulouse. Colloque international - La recherche en éducation relative à l'environnement : bilan, enjeux et perspectives. 5 et 6 novembre 1997, Université du Québec à Montréal, Montréal.

De Landsheere, V. (1992). L'éducation et la formation. Paris : Presses Universitaires de France. 
De Melo, J.C. (1997). Le site-école communauté à « Pedra 90 », Cuiabá, Mato Grosso : caractéristiques et perspectives en ERE. Colloque international - La recherche en éducation relative à l'environnement : bilan, enjeux et perspectives. 5 et 6 novembre 1997, Université du Québec à Montréal, Montréal.

Desautels, J. (1997). Rapport au savoir et éducation relative à l'environnement. Colloque international - La recherche en éducation relative à l'environnement : bilan, enjeux et perspectives. 5 et 6 novembre 1997, Université du Québec à Montréal, Montréal.

Desroches, F. (1997). Une éducation ouverte sur le monde. Colloque international - La recherche en éducation relative à l'environnement : bilan, enjeux et perspectives. 5 et 6 novembre 1997, Université du Québec à Montréal, Montréal.

Dionne, L. (1997). Conceptions, croyances et pratiques en matière d'éducation à l'environnement chez les éducateurs du primaire en République de Guinée. Mémoire de maîtrise en éducation, Université du Québec à Montréal.

Dubé, S. (1997). L'éducation relative à l'environnement dans une perspective de partenariat écolecommunauté. Rapport de recherche de maîtrise en sciences de l'environnement, Université du Québec à Montréal.

Dubé, S. et Orellana, I. (1997). Bilan et perspectives de l'éducation relative à l'environnement au Québec. Montréal : AQPERE.

El Hattab, A. (1997). Les représentations discursives d'enseignants des sciences en contexte marocain à l'égard de la nature du savoir écologique et sociale de sa production. Colloque international - La recherche en éducation relative à l'environnement : bilan, enjeux et perspectives. 5 et 6 novembre 1997, Université du Québec à Montréal, Montréal.

Elliott, J. (1994). Le développement d'une éducation relative à l'environnement à partir de la collectivité par la recherche-action. Dans OCDE (dir.), Évaluer l'innovation dans l'éducation à l'environnement. Paris : Organisation de coopération et de développement économiques. p. 33-65.

Fauteux, S. (1997). La communication intersubjective liée aux pratiques de l'ERE. Colloque international - La recherche en éducation relative à l'environnement : bilan, enjeux et perspectives. 5 et 6 novembre 1997, Université du Québec à Montréal, Montréal.

Ferrer, C. (1997). Un modèle d'éducation dans une perspective planétaire dans la formation d'enseignants. Colloque international - La recherche en éducation relative à l'environnement : bilan, enjeux et perspectives. 5 et 6 novembre 1997, Université du Québec à Montréal, Montréal.

Fontaine, S. (1994). Recherche en éducation et changement. Dans J. Chevrier (dir.), La recherche en éducation. Montréal : Les Éditions Logiques. p. 43-59.

Garnier, C. (1997). Éléments fondamentaux de la théorie des représentations sociales : une application à la santé et à l'environnement. Colloque international - La recherche en éducation relative à l'environnement : bilan, enjeux et perspectives. 5 et 6 novembre 1997, Université du Québec à Montréal, Montréal.

Gauthier, B. (1997). Modèle pédagogique réflexif basé sur la recherche-action mis à l'essai en milieu culturel africain. Colloque international - La recherche en éducation relative à

l'environnement : bilan, enjeux et perspectives. 5 et 6 novembre 1997, Université du Québec à Montréal, Montréal.

Gauthier, V. (1997). L'éducation dans une perspective mondiale par l'approche systémique et holistique. Colloque international - La recherche en éducation relative à l'environnement : bilan, enjeux et perspectives. 5 et 6 novembre 1997, Université du Québec à Montréal, Montréal. 
Goffin, L. (1998). L'environnement comme éco-sociosystème. Dans M. Loriaux (dir.), Populations et développement : une approche globale et systémique. Louvain-la-Neuve et Paris : Academia-Bruylant et l'Harmattan. p. 199-230.

Goffin, L. (1997). Le lien recherche-formation-intervention en éducation relative à l'environnement. Colloque international - La recherche en éducation relative à l'environnement : bilan, enjeux et perspectives. 5 et 6 novembre 1997, Université du Québec à Montréal, Montréal.

Gough, A. (1997). Education and the environment. Policy, trends and the problems of marginalisation. Melbourne (Victoria) : The Australian Council for Educational Research.

Grodya, D. (1997). La radio communautaire au Québec en matière d'éducation relative à l'environnement - Rôle actuel et potentiel. Rapport de recherche de maîtrise en sciences de l'environnement, Université du Québec à Montréal.

Guba, E.G. (1990). The alternative paradigm dialog. Dans E.G. Guba (dir.), The paradigm dialog. Newbury Park, Californie : Sage Publications. p. 17-27.

Guilbert, L. (1997). L'émergence d'un nouveau paradigme : la réflexivité et la pensée critique dans une perspective renouvelée en ERE. Colloque international - La recherche en éducation relative à l'environnement : bilan, enjeux et perspectives. 5 et 6 novembre 1997, Université du Québec à Montréal, Montréal.

Habermas, J. (1974). Theory and practice. London : Heinemann.

Hart, P. (1993). Perspectives in environmental education research : Paradigm of critical reflective inquiry. Dans R. Mrazek (dir.), Alternative paradigms in environmental education research. Troy, Ohio : North American Association for Environmental Education. p. 107-131.

Hart, P. (1991). Thoughts on research paradigms in EE. Environmental Communicator. JanvierFévrier 1991. North American Association for Environmental Education, p. 5

Hungerford, H. et Volk, T. (1990). Changing learner behavior through environmental education, The Journal of Environmental Education, 21(3), 8-21.

Hugon, M.A. et Siebel, C. (1988). Recherches impliquées - Recherche-action : le cas de l'éducation. Bruxelles : De Boeck-Wesmail.

Janse van Rensburg, E. (1994). Social transformation in response to the environmental crisis : the role of education and research. Australian Journal of Environmental Education, 10, 1-20.

Lalonde, C. (1997). L'art et l'ERE pour une éducation globale. Mémoire de maîtrise en éducation, Université du Québec à Hull.

Langevin, L. (1997). L'animation pédagogique et l'éducation à distance dans une démarche collaborative. Le cas du projet EDAMAZ. Colloque international - La recherche en éducation relative à l'environnement : bilan, enjeux et perspectives. 5 et 6 novembre 1997, Université du Québec à Montréal, Montréal.

Laurent, D. (1997). L'ERE : une condition nécessaire, mais non suffisante du développement durable. Colloque international - La recherche en éducation relative à l'environnement : bilan, enjeux et perspectives. 5 et 6 novembre 1997, Université du Québec à Montréal, Montréal.

Macchabée, L. (1997). La construction sociale de la représentation de l'environnement au processus de restauration des écosystèmes. Colloque international - La recherche en éducation relative à l'environnement : bilan, enjeux et perspectives. 5 et 6 novembre 1997, Université du Québec à Montréal, Montréal. 
Marquis, G. (1997). Éducation relative à l'environnement et développement. Colloque international - La recherche en éducation relative à l'environnement : bilan, enjeux et perspectives. 5 et 6 novembre 1997, Université du Québec à Montréal, Montréal.

Mayer, M. (1997). Action research and the production of knowledge : the experience of international project on environmental education. Dans S. Hollongsworth (dir.), Internal action research: A casebook for educational reform. London : The Falmer Press, p. 112-123.

Morin, A. (1992). Recherche-action intégrale et participation coopérative. vol. 1 et 2. Montréal : Agence d'Arc.

Orellana, I. (1997). La notion de communauté d'apprentissage en ERE. Colloque international - La recherche en éducation relative à l'environnement : bilan, enjeux et perspectives. 5 et 6 novembre 1997, Université du Québec à Montréal, Montréal.

Pelletier, M. et Lefebvre, N. (1997). De l'ERE pour survivre : analyse d'un modèle d'intervention dans une petite école de milieu rural. Colloque international - La recherche en éducation relative à l'environnement bilan, enjeux et perspectives. 5 et 6 novembre 1997, Université du Québec à Montréal, Montréal.

Picard, M. (1995). Étude des représentations sociales de l'environnement chez des enseignantes du primaire. Mémoire de maîtrise en éducation, Université du Québec à Rimouski.

Ponton, M. (1998). Étude des représentations sociales des jeunes à l'égard de l'environnement. Projet de thèse de doctorat en éducation, Université de Sherbrooke.

Ponton, M. (1997). Contribution des représentations sociales en ERE : diagnostic et perspectives. Colloque international - La recherche en éducation relative à l'environnement : bilan, enjeux et perspectives. 5 et 6 novembre 1997, Université du Québec à Montréal, Montréal.

Poudrier, C. (1996). Expérimentation en milieu scolaire québécois du modèle pédagogique de William B. Stapp : La résolution de problèmes communautaires. Rapport de recherche, Université du Québec à Trois-Rivières.

Pruneau, D. et Chouinard, O. (1997). Un modèle d'intervention qui favorise la relation personne groupe social - environnement. Dans M. Hrimech et F. Jutras (dir.), Défis et enjeux de l'éducation dans une perspective planétaire. Sherbrooke : Éditions du CRP. p. 107-127.

Robertson, A. (1994). Toward constructivist research in environmental education. The Journal of Environmental Education, 25(2), 21-31.

Robottom, I. et Hart, P. (1995). Behaviorist EE research : Environmentalism as Individualism. The Journal of Environmental Education, 26(2), 5-9.

Robottom, I. et Hart, P. (1993). Research in environmental education. Deakin, Australie : Deakin van De

Rooney, E. (1997). Du comportement à la conduite, plus qu'un simple jeu de mots ! Colloque international - La recherche en éducation relative à l'environnement : bilan, enjeux et perspectives. 5 et 6 novembre 1997, Université du Québec à Montréal, Montréal.

Roy, A. (1997). Une étude multicas sur les théories personnelles et pratiques éducatives relatives aux valeurs environnementales. Colloque international - La recherche en éducation relative à l'environnement : bilan, enjeux et perspectives. 5 et 6 novembre 1997, Université du Québec à Montréal, Montréal.

Roy, L. (1997). Modèle réflexif pour l'animation et la création d'activités éducatives traitant des enjeux environnementaux. Colloque international - La recherche en éducation relative à 
l'environnement : bilan, enjeux et perspectives. 5 et 6 novembre 1997, Université du Québec à Montréal, Montréal.

Saint-Arnaud, Y. (1992). Connaître par l'action. Montréal : Les Presses de l'Université de Montréal. Santoire, M.-J. (1999). Étude descriptive de la recherche francophone en éducation relative à l'environnement au Québec et dans l'Est du Canada. Rapport de recherche de maîtrise en sciences de l'environnement, Université du Québec à Montréal.

Sauvé, L. (1998). L'éducation relative à l'environnement, entre modernité et postmodernité - Les propositions du développement durable et de l'avenir viable. Canadian Journal of Environmental Education, 4, Printemps 1999.

Sauvé, L. (1997 a). Pour une éducation relative à l'environnement. 2e édition. Montréal : Guérin.

Sauvé, L. (1997 b). Pour une recherche critique en éducation relative à l'environnement. Dans C. Baudoux et M. Anadon (dir.), La recherche en éducation, la personne et le changement social. Les Cahiers du LABRAPS - Laboratoire de recherche en administration et politiques scolaires, Université Laval, vol. 23. p. 103-122.

Sauvé, L. (1997 c). L'approche critique en éducation relative à l'environnement : origines théoriques et applications à la formation des enseignants. Revue des sciences de l'éducation, XXIII (1), 169-189.

Sauvé, L. et Garnier, C. (1999). Une phénoménographie de l'environnement - Réflexions théoriques et méthodologiques sur l'analyse des représentations sociales. Dans M. Rouquette et C. Garnier (dir.), Représentations sociales et éducation. Bruxelles : DeBoeck. (Accepté pour publication).

Sauvé, L. et Bégin, R. (1997). ERE et enseignement des sciences : représentations et pratiques. Colloque international - La recherche en éducation relative à l'environnement : bilan, enjeux et perspectives. 5 et 6 novembre 1997, Université du Québec à Montréal, Montréal.

Sauvé, L. et collaborateurs. (1997). L'éducation relative à l'environnement à l'école secondaire québécoise - Éléments de diagnostic. Montréal : CIRADE.

Savoie-Zajc, L. (1997). De la recherche, activité d'expertise à la recherche comme stratégie de changement : un outil de transformation des pratiques enseignantes dans le cadre du projet EDAMAZ. Colloque international - La recherche en éducation relative à l'environnement : bilan, enjeux et perspectives. 5 et 6 novembre 1997, Université du Québec à Montréal, Montréal.

Schön, D. (1987). Educating the reflective practitionner. San Francisco : Josey-Bass.

ouchon, C. et Raichvarg, D. (1997). À la recherche d'une formation spécifique à l'ERE dans une perspective d'éducation à la citoyenneté. Colloque international - La recherche en éducation relative à l'environnement : bilan, enjeux et perspectives. 5 et 6 novembre 1997, Université du Québec à Montréal, Montréal.

Sow, M. (1996). Design et expérimentation d'un programme de formation des enseignants de l'élémentaire de Guinée en éducation relative à l'environnement. Mémoire de maitrise en sciences de l'environnement, Université du Québec à Montréal.

Taelman, P. (1997). Évaluation de projets d'ERE pour l'octroi de subventions publiques : réflexions, propositions méthodologiques et perspectives. Colloque international - La recherche en éducation relative à l'environnement : bilan, enjeux et perspectives. 5 et 6 novembre 1997, Université du Québec à Montréal, Montréal. 
Tessier, R. (1997). L'analyse des éthiques en présence dans un dossier environnemental : un atout pour l'ERE. Colloque international - La recherche en éducation relative à l'environnement : bilan, enjeux et perspectives. 5 et 6 novembre 1997, Université du Québec à Montréal, Montréal.

Toussaint, R.M.J., Leclerc, M.-L., Samson, G., Céran, R. et Pellaud, F. (1997). Actions et recherches dans un partenariat nord-sud. ERE et développement durable. Colloque international - $L a$ recherche en éducation relative à l'environnement : bilan, enjeux et perspectives. 5 et 6 novembre 1997, Université du Québec à Montréal, Montréal.

Vaillancourt, J.-G. (1997). Repenser l'ERE dans la perspective du développement durable. Colloque international - La recherche en éducation relative à l'environnement : bilan, enjeux et perspectives. 5 et 6 novembre 1997, Université du Québec à Montréal, Montréal.

Van der Maren, J.M. (1995). Méthodes de recherche pour l'éducation. Montréal et Bruxelles : Les Presses de l'Université de Montréal et De Boeck Université.

Walker, K. (1997). Challenging critical theory in environmental education. Environmental Education Research, 3(2), 155-162.

Wals, A. (1990). What you can't measure still exists. Environmental Communicator, novembredécembre 1990, North American Association for Environmental Education, p. 12.

Wolf-Michael, R. (1997). Vers une désinstitutionnalisation des sciences à l'école : la prise en compte du milieu. Colloque international - La recherche en éducation relative à l'environnement : bilan, enjeux et perspectives. 5 et 6 novembre 1997, Université du Québec à Montréal, Montréal

\section{NOTES}

1. Ce colloque a été organisé dans le cadre des activités du CIRADE (Centre interdisciplinaire de recherche sur l'apprentissage et le développement en éducation) et de l'ISE (Institut des sciences de l'environnement) de l'Université du Québec à Montréal. Le comité organisateur était composé de Lucie Sauvé, Catherine Garnier (directrice du CIRADE) et Isabel Orellana (doctorat en éducation).

2. Une analyse des perspectives complémentaires selon lesquelles il est possible d'aborder l'ERE est présentée dans Sauvé (1997 a, p. 19-26).

3. Je tiens à souligner le grand intérêt du patient travail de Marie-Josée Santoire, étudiante à la maîtrise en sciences de l'environnement de l'UQAM. La première exploration des productions de recherche qu'elle a réalisée, avec rigueur et vision critique, fournit des pistes très inspirantes pour la poursuite de la recherche en ce sens et facilitera la construction d'une méthodologie appropriée pour des analyses plus approfondies.

4. Cette notion de recherche-évaluation sera explicitée par Goffin et Sauvé (1999) dans le prochain volume de cette revue.

5. Tel que signalé, un projet de répertoire a été associé aux activités du colloque. Des fiches d'inscription des recherches à ce répertoire ont été acheminées aux chercheurs de la francophonie, en même temps que l'invitation à participer au colloque. Soixante-douze (72) fiches nous sont parvenues, dont soixante et une (61) du Québec et de l'Est du Canada. La proximité de l'événement et les stratégies de rappel expliquent que davantage de fiches nous sont parvenues de chercheurs nord-américains. C'est d'ailleurs cette région géographique du Québec et de l'Est du Canada qui a été retenue pour l'étude de Marie-Josée Santoire (1999). Cette étude a considéré les 61 fiches reçues de même que les 12 productions de recherche repérées en bibliothèque au Québec, pour un total de 73 recherches. Si l'on ajoute à ces 73 productions, les 11 fiches provenant d'Europe ou d'Afrique, nous obtenons un total de 84 recherches. 


\section{RÉSUMÉS}

Le Colloque international francophone sur la recherche en éducation relative à l'environnement (ERE), tenu à Montréal, en novembre 1997, a été l'occasion de faire un bilan descriptif et critique des activités de recherche en ERE au sein de la francophonie et de poursuivre la réflexion sur les enjeux et perspectives d'une telle recherche. Bien que peu diffusée et rarement discutée, la recherche en ERE produite en langue française apparaît déjà relativement riche et diversifiée. À part le constat de l'importance récemment accordée au courant de la recherche sur les représentations sociales, l'exploration des activités et des productions de recherche francophones en ERE conduit aux mêmes observations et suscite les mêmes questions que pour l'ensemble de la recherche en ERE. En particulier, il importe de définir la spécificité de la recherche en ce domaine, de développer des outils conceptuels pour la caractériser, d'en évaluer la pertinence et d'identifier des voies de développement. Cet article propose quelques réflexions et observations sur ces questions.

The international Colloquium La recherche en éducation relative à l'environnement : bilan, enjeux et perspectives, held in Montréal in November 1997, was an opportunity to describe and critically assess environmental education (EE) research activities among French speaking communities and to pursue the reflection on the issues and perspectives of such a research. Although they have been poorly disseminated and rarely discussed, EE research activities produced in French appear to already be relatively rich and diversified. An exploration of these research activities leads to a number of observations and questions. Among others, it appears important to define the specificity of research in this field, to develop conceptual tools to characterize it, to evaluate its relevance and to identify further means of development. This paper proposes some reflections on these topics.

\section{AUTEUR}

\section{LUCIE SAUVÉ}

Lucie Sauvé est professeure au département des sciences de l'éducation de l'Université du Québec à Montréal. Au sein de cette institution, elle est également chercheure affiliée au CIRADE (Centre interdisciplinaire de recherche sur l'apprentissage et le développement en éducation) et membre de l'Institut des sciences de l'environnement. Elle assume la responsabilité du Programme court en éducation relative à l'environnement de l'UQAM et coordonne des projets de coopération internationale en ERE. 